\title{
When Professional Judgment Conflicts with the Regulations
}

It was obvious to everyone that Jenn Cotter had made a good choice with Mike Reiss, her new veterinary resident. Nevertheless, Reiss was wise enough to know that he still had a lot to learn. This insight was confirmed with an incident that did not result in any animal pain or suffering, but certainly caused Reiss considerable distress.

Reiss performed a relatively harmless procedure on Toots, a two-year-old macaque. He had performed the procedure frequently on other monkeys without any problems. Using light chemical restraint and a long-acting local anesthetic for pain control, he made a $1.5-\mathrm{cm}$ incision on the side of her chest and placed a drug-filled capsule into the incision. About $2 \mathrm{~h}$ later, as the anesthetic was wearing off, he gave her a dose of butorphanol for additional pain relief, which was the same analgesia regimen he had used on all the other monkeys. The animals almost never bothered with the incision, never received more butorphanol, and all healed uneventfully.

Shana Madela, the USDA veterinary officer, was reviewing the IACUC protocol that included the study involving Toots. She had already seen Toots and the other macaques, all of which seemed to be doing fine. Madela noticed that the IACUC had approved the use of butorphanol every $4 \mathrm{~h}$ for the first $12 \mathrm{~h}$, then as needed. Nevertheless, Reiss had clearly recorded using it just once on Toots and the other monkeys. Madela asked for an explanation, and Reiss responded that in his clinical judgment one dose provided adequate analgesia. This was confirmed, he said, by their excellent healing and by the observation that the monkeys almost never picked at the incision. Madela was polite but told Reiss that, if he wanted to change the approved procedure, the Principal Investigator (PI) should have amended the protocol. Reiss was astonished that another veterinarian was challenging his clinical judgment, particularly when the results seemed to confirm the accuracy of his judgment. Madela told Reiss that she certainly was not challenging his judgment but was only informing him that changes in analgesic needs must appear as modifications of the protocol.

In her inspection report, Madela indicated that there was an infraction of Animal Welfare Act (AWA) regulation $\$ 2.31(\mathrm{e})(3)$, which requires a complete description of the proposed animal use. Reiss was confounded about the proper course of action, so he sought counsel from Cotter, who quickly opened her copy of the regulations and cited $\$ 2.40$ (a)(5), which calls for adequate preprocedural and postprocedural care in accordance with established veterinary medical and nursing procedures. She argued with Madela that established veterinary medical procedures do not call for the use of drugs when they are not needed. Once again, Madela said that she was not challenging Reiss' judgment, but that the protocol should have been amended to reflect his judgment.

A stalemate was developing, because Madela and Cotter were not willing to concede their positions. Although there would be opportunity to provide a written response to any USDA citation, at this point in time do you think Madela or Cotter had the stronger argument?

\section{An Amendment Is in Order}

Marge J. Piel, MS, DVM, DipACLAM and Christine C. Lieggi, DVM

In this situation, Madela has recognized that the submitted description of the proposed animal use was not followed by the veterinarian exactly as written. The AWA requires a complete accounting of animal use, with the expectation that the IUCUC-approved protocol will be followed. In this case, Reiss, the veterinarian responsible for performing the procedures and for postprocedural care, considers the use of butorphanol every $4 \mathrm{~h}$ to be excessive and unnecessary. Believing the animals to be free of pain, he decided that additional injections, which themselves may be painful, were unnecessary. Cotter supports her resident's opinion, citing that adequate veterinary care, according to AWA $\$ 2.40$ (a)(5), is required to be in accordance with established veterinary medical procedures, and hence the administration of unnecessary drugs is not justified.

In this instance, Reiss is not acting as clinical veterinarian but rather is providing a service-implanting drug delivery capsules for the investigator-and therefore is functioning as an extension of the investigator. As such, he must follow the IACUC-approved protocol as described. When he first discovered that the approved analgesic protocol was excessive, he should have approached Cotter to discuss the regimen and then asked the investigator to submit a protocol modification to the IACUC to amend the postoperative analgesic regimen. The modification should have included the option to alter the frequency of administration by including the phrase 'as needed for pain', which should be followed by criteria for use in determining the conditions for the need of additional analgesics depending on each individual animal's needs.

The larger problem is that the appropriateness of the analgesic regimen should have been discussed during review of the protocol when it was first submitted, either through a veterinary prereview or during the 